\title{
A Family of Joint Possibility Distributions for Adding Interactive Fuzzy Numbers Inspired by Biomathematical Models
}

\author{
Estevão Esmi ${ }^{*}$ Gustavo Barroso ${ }^{\ddagger}$ Laécio C. Barros ${ }^{\ddagger}{\text { Peter Sussner }{ }^{\ddagger}}^{\natural}$ \\ ${ }^{1}$ University of Campinas, Department of Applied Mathematics, Campinas, SP 13083-859, Brazil
}

\begin{abstract}
In many models for real-world problems such as the law of conservation of energy, the sum of parameters must be zero. In this paper, we investigate the problem of adding fuzzy numbers using the extension principle based on the concept of the joint possibility distribution. In particular, we present a formula to produce a family of joint possibility distribution indexed by a parameter $\gamma$ for a given pair of fuzzy numbers, say $A_{1}$ and $A_{2}$, such that the norm of the corresponding sums of $A_{1}$ and $A_{2}$ form a increasing mapping with respect to $\gamma$.
\end{abstract}

Keywords: Biomathematical models, sup-J extension, fuzzy numbers, sum operation.

\section{Introduction}

The law of conservation naturally appears in many biomathematical models. For example, mathematical models of disease transmission in a short period of time assumes that there are neither births nor death. This assumption is described by $S+I=1$, where $S$ and $I$ stands for the rates of susceptible and infected populations, respectively, in every instance of time. Assuming that the model is subject to uncertainty, Baetens and Baets treated $S$ and $I$ as interactive fuzzy numbers in order to study the evolution of epidemics over time and space [1]. In this context, Cabral and Barros [2] considered the interactivity between contact rates and the initial infected population. The same authors also considered the interactivity between the decay rate $(w)$ and the initial condition $\left(x_{0}\right)$ in a Malthusian decay model such that $w+x_{0}=2$. Note that the analysis of the dynamics of a given model depends on how this interactivity is mathematically expressed. The last comment motivated us to develop novel approaches for adding interactive fuzzy numbers that allow us to remain consistent with these underlying conservation assumptions.

*This work was partially supported by Fapesp under grant n. $2014 / 10067-8$

${ }^{\dagger}$ This work was partially supported by $\mathrm{CNPq}$ under grant n. $132765 / 2014-4$

¥This work was partially supported by CNPq under grant n. $305862 / 2013-8$

$\S$ This work was partially supported by CNPq under grant n. $311695 / 2014-0$

\section{Joint Possibility Distribution and Extension Principle}

A fuzzy set $A$ over a universal set $X$ is determined by its membership function [3] $\mu_{A}: X \rightarrow[0,1]$ where $\mu_{A}(x)$ yields the membership degree of $x$ in $A$. For notational convenience, we simply write $A(x)$ instead of $\mu_{A}(x)$ for all $x \in X$. The symbol $\mathcal{F}(X)$ denotes the class of fuzzy sets over the universal set $X$. The total order relation on $[0,1]$ induces the partial order relation given by the inclusion operator $\subseteq$ on $\mathcal{F}(X)$ as follows: $A \subseteq B \Leftrightarrow A(x) \leq B(x)$ for all $x \in X$. Moreover, the usual operations of infimum and supremum of the complete lattice $[0,1][4]$, denoted respectively by $\Lambda$ and $\bigvee$, induce the usual intersection and union operations for fuzzy sets.

The total order relation on $[0,1]$ with the usual operations of infimum and supremum, denoted respectively by $\Lambda$ and $\bigvee$, induces the partial order relation given by the inclusion operator $\subseteq$ on $\mathcal{F}(X)$ as follows: $A \subseteq B \Leftrightarrow A(x) \leq B(x)$ for all $x \in X$. Note that the partially ordered set $\mathcal{F}(X)$ represents a complete lattice [4].

Operators of fuzzy logic arises from the generalization of operators of Boolean logic to the unit interval $[0,1]$. Here, we will only employ extensions of the "and" connector known as t-norms. Mathematically speaking, a t-norm is a commutative, associative, and increasing function $t:[0,1]^{2} \rightarrow[0,1]$ with identity element 1 .

The power set of $X$, denoted by $\mathcal{P}(X)$, can be embedded into $\mathcal{F}(X)$ since every subset $Y$ of $X$ is uniquely determined by its characteristic function $\chi_{Y}: X \rightarrow\{0,1\} \subseteq[0,1]$. The concept of a relation can also be easily extended to the fuzzy domain as follows. A fuzzy relation between an arbitrary set $X$ and another arbitrary set $Y$ is given by a fuzzy set in $X \times Y$, that is, a function $R: X \times Y \rightarrow[0,1]$. The value $R(x, y)$ can be interpreted as the degree of relationship between $x$ and $y$ for $(x, y) \in X \times Y$ [5].

Two fuzzy relations can be combined by means of a fuzzy relational composition in order to produce another fuzzy relation. Here we only employ fuzzy relational compositions of the type sup- $t$ that are defined as follows. Let $R \in \mathcal{F}(X \times Y)$ and $S \in \mathcal{F}(Y \times Z)$, where $X, Y$, and $Z$ are arbitrary universes, and let $t$ be a t-norm. The sup- $t$ composition of $R$ and $S$ is the following fuzzy relation 
$R \circ_{t} S \in \mathcal{F}(X \times Z)[6]:$

$\left(R \circ_{t} S\right)(x, z)=\bigvee_{y \in Y} R(x, y) t S(y, z) \forall(x, z) \in X \times Z$.

Similarly, the sup- $t$ composition of a fuzzy relation $R \in \mathcal{F}(X \times Y)$ and a fuzzy set $S \in \mathcal{F}(Y)$ yields the following fuzzy set $R \circ_{t} S \in \mathcal{F}(X)$ [6]:

$$
\left(R \circ_{t} S\right)(x)=\bigvee_{y \in Y} R(x, y) t S(y) \forall x \in X .
$$

If $t$ equals $\wedge$, i.e., the minimum t-norm, in Equations (1) and (2), then we simply use the symbol $\circ$ instead of $\mathrm{o}_{t}$.

A fuzzy set $R$ in an n-dimensional domain $X=$ $X_{1} \times \ldots \times X_{n}$ can also be interpreted as an n-ary fuzzy relation [7]. The projection of a fuzzy relation $R \in \mathcal{F}\left(X_{1} \times \ldots \times X_{n}\right)$ onto $X_{i}$, where $1 \leq i \leq n$, is the fuzzy set $\Pi_{R}^{i}$ of $X_{i}$ given by $[6,7]$ :

$$
\Pi_{R}^{i}(y)=\bigvee\left\{R(\mathbf{x}) \mid \mathbf{x} \in X \text { with } x_{i}=y\right\} \forall y \in X_{i}
$$

A fuzzy set $A$ of $X$ can be identified with its $\alpha$ cuts $[6,8]$. Given $\alpha \in(0,1]$, the $\alpha$-cut of $A$ is defined by $[A]^{\alpha}=\{x \in X \mid A(x) \geq \alpha\}$. If $X$ is also a topological space, then $[A]^{0}$ is defined as the closure of the support of $A$, that is, $\operatorname{cl}\{x \mid A(x)>0\}$. The following properties hold for all $A, B \in \mathcal{F}(X)$ :

(P1) $A=B \Leftrightarrow[A]^{\alpha}=[B]^{\alpha}$ for all $\alpha \in(0,1]$;

(P2) $A \subseteq B \Leftrightarrow[A]^{\alpha} \subseteq[B]^{\alpha}$ for all $\alpha \in(0,1]$;

(P3) for every $x \in X$, we have that

$$
A(x)=\bigvee\left\{\alpha \in(0,1] \mid x \in[A]^{\alpha}\right\} .
$$

Let $X_{1}, \ldots, X_{n}$, and $Y$ be arbitrary universes. Zadeh's extension principle [9] is a mathematical tool for extending any function $f: X_{1} \times \ldots \times X_{n} \rightarrow$ $Y$ to a function $f_{M}: \mathcal{F}\left(X_{1}\right) \times \ldots \times \mathcal{F}\left(X_{n}\right) \rightarrow \mathcal{F}(Y)$. Recall that $f_{M}$ is defined by

$f_{M}\left(A_{1}, \ldots, A_{n}\right)(z)=\bigvee_{\mathbf{x} \in f^{-1}(z)} A_{1}\left(x_{1}\right) \wedge \ldots \wedge A_{n}\left(x_{n}\right)$,

where $f^{-1}(z)=\left\{\left(x_{1}, \ldots, x_{n}\right) \in X_{1} \times \ldots \times\right.$ $\left.X_{n} \mid f\left(x_{1}, \ldots, x_{n}\right)=z\right\}$. Note that $f^{-1}(z)$ can be empty, in which case we have that $f_{M}\left(A_{1}, \ldots, A_{n}\right)(z)=0$.

For the case where $f: \mathbb{R}^{n} \rightarrow \mathbb{R}$, Fuller and Majlender $[10,11]$ employed the notion of a joint possibility distribution to propose a method that deals with an n-tuple of fuzzy numbers and that can be viewed as a generalization of Zadeh's extension principle. Recall that a fuzzy number is a fuzzy set of $\mathbb{R}$, i.e., an element of $\mathcal{F}(\mathbb{R})$, that is normal, convex, and upper semi-continuous and has a compact 0cut. The set of fuzzy numbers of $\mathbb{R}$ is denoted using the symbol $\mathbb{R}_{\mathcal{F}}$. Typical examples of fuzzy numbers include triangular and trapezoidal fuzzy sets. The next proposition characterizes the $\alpha$-cuts of a fuzzy number $[12,8]$.
Proposition 1 Let $A \in \mathbb{R}_{\mathcal{F}}$. If $M^{\alpha}=[A]^{\alpha}$ for $\alpha \in$ $[0,1]$, then the family of intervals $\left\{M^{\alpha} \mid \alpha \in[0,1]\right\}$ has the following properties:

1. $M^{\alpha}$ is a non-empty closed interval of $\mathbb{R}$ for all $\alpha \in[0,1]$;

2. $M^{\alpha} \subseteq M^{\beta}$ for $0 \leq \beta \leq \alpha \leq 1$;

3. for any sequence $\left(\alpha_{n}\right)$ that converges from below to $\alpha \in(0,1]$, we have that

$$
\bigcap_{n=1}^{\infty} M^{\alpha_{n}}=M^{\alpha}
$$

4. for any sequence $\left(\alpha_{n}\right)$ that converges from above to 0 , we have that

$$
c l\left(\bigcup_{n=1}^{\infty} M^{\alpha_{n}}\right)=M^{0}
$$

The norm of a fuzzy number is defined as the function $\|\cdot\|: \mathbb{R}_{\mathcal{F}} \rightarrow[0, \infty)$ given by: [12]

$$
\|A\|=\bigvee_{\alpha \in[0,1]} \bigvee_{x \in[A]^{\alpha}}|x|, \quad \forall A \in \mathbb{R}_{\mathcal{F}} .
$$

Definition 1 A fuzzy relation $J \in \mathcal{F}\left(\mathbb{R}^{n}\right)$ is said to be a joint possibility distribution of $A_{1}, \ldots, A_{n} \in$ $\mathbb{R}_{\mathcal{F}}$ if

$$
A_{i}(x)=\Pi_{J}^{i}(x)
$$

holds for all $x \in \mathbb{R}$ and $i=1, \ldots, n$.

Furthermore, $J$ is called non-interactive if

$$
J\left(x_{1}, \ldots, x_{n}\right)=\bigwedge_{i=1}^{n} A_{i}\left(x_{i}\right)
$$

for all $\left(x_{1}, \ldots, x_{n}\right) \in \mathbb{R}^{n}$.

Equation (6) ensures that $J\left(x_{1}, \ldots, x_{n}\right) \leq A_{i}\left(x_{i}\right)$ for all $i=1, \ldots, n$, which implies that any joint possibility distribution $J$ of $A_{1}, \ldots, A_{n}$ satisfies the inequality $J\left(x_{1}, \ldots, x_{n}\right) \leq \bigwedge_{i=1}^{n} A_{i}\left(x_{i}\right)$. In other words, any joint possibility distribution $J$ of $A_{1}, \ldots, A_{n}$ is contained in the non-interactive joint possibility distribution of $A_{1}, \ldots, A_{n}$.

Definition 2 ([10]) Let $J \in \mathcal{F}\left(\mathbb{R}^{n}\right)$ be a joint possibility distribution of $A_{1}, \ldots, A_{n} \in \mathbb{R}_{\mathcal{F}}$ and let $f: \mathbb{R}^{n} \rightarrow \mathbb{R}$. The sup-J extension of $f$ is defined by

$$
f_{J}\left(A_{1}, \ldots, A_{n}\right)(y)=\bigvee_{\left(x_{1}, \ldots, x_{n}\right) \in f^{-1}(y)} J\left(x_{1}, \ldots, x_{n}\right)
$$

for all $A_{1}, \ldots, A_{n} \in \mathbb{R}_{\mathcal{F}}$.

Note that if $J$ corresponds to the non-interactive joint possibility distribution of $A_{1}, \ldots, A_{n}$ given by Equation (7), then $f_{J}\left(A_{1}, \ldots, A_{n}\right)$ boils down to Zadeh's extension of $f$ at $\left(A_{1}, \ldots, A_{n}\right)$. Moreover, if $J\left(x_{1}, \ldots, x_{n}\right)=A_{1}\left(x_{1}\right) t \ldots t A_{n}\left(x_{n}\right)$ for all $\left(x_{1}, \ldots, x_{n}\right) \in \mathbb{R}^{n}$ and for some fixed t-norm $t$, then $f_{J}\left(A_{1}, \ldots, A_{n}\right)$ is said to be the t-norm based extension principle of $f[13,14]$. 
The fuzzy set $f_{J}\left(A_{1}, \ldots, A_{n}\right)$ can also be expressed in terms of a fuzzy relational composition as follows:

$$
R_{f} \circ J=f_{J}\left(A_{1}, \ldots, A_{n}\right)
$$

where $R_{f}$ is the fuzzy set of $\mathbb{R} \times \mathbb{R}^{n}$ whose membership function is given by

$$
R_{f}(y, \mathbf{x})= \begin{cases}1 & \text { if } y=f(\mathbf{x}) \\ 0 & \text { otherwise }\end{cases}
$$

for all $y \in \mathbb{R}$ and for all $\mathbf{x}=\left(x_{1}, \ldots, x_{n}\right) \in \mathbb{R}^{n}$.

Fuller and Majlender also introduced the concept of completely (positively/negatively) correlated fuzzy numbers in terms of a given joint possibility distribution. Specifically, if $J$ is a joint possibility distribution of two fuzzy numbers $A$ and $B$, then $A$ and $B$ are said to be completely correlated if there exist $q, r \in \mathbb{R}$ with $q \neq 0$ such that $J$ equals $J_{q, r}$ where

$$
\begin{aligned}
J_{q, r}(x, y)= & A(x) \chi_{\{q x+r=y\}}(x, y) \\
= & B(y) \chi_{\{q x+r=y\}}(x, y) \\
& \forall x, y \in \mathbb{R} .
\end{aligned}
$$

Recall that $\chi_{\{q x+r=y\}}$ is the characteristic function of the the subset $\{(x, q x+r) \mid x \in \mathbb{R}\}$ of $\mathbb{R}^{2}$, that is

$$
\chi_{\{q x+r=y\}}(x, y)=\left\{\begin{array}{ll}
1 & \text { if } q x+r=y \\
0 & \text { otherwise }
\end{array} .\right.
$$

Moreover, we speak of completely positively (or negatively) correlated fuzzy numbers if $q>0$ (or $q<0$ ).

Note that the t-norm-based approach to construct joint possibility distributions is defined for any finite set of fuzzy numbers whereas the Fuller and Majlender's approach is only defined for certain pairs of fuzzy numbers. In general, a triangular fuzzy number and a trapezoidal fuzzy number can not be completely correlated.

The notion of interactivity between two fuzzy numbers only arises if a joint possibility distribution is at hand. Thus, the task of defining an interactivity relation for two fuzzy numbers is tied to the one of determining a joint possibility distribution. Therefore, developing appropriate formulas for constructing joint possibility distributions for two or more given fuzzy numbers is essential for tuning the resulting interactivity relations. In Section 4, we will provide some formulas for constructing joint possibility distributions for any pair of fuzzy numbers. Note that the choice of a joint possibility distribution determines if some given fuzzy numbers are interactive or not.

\section{Addition of Interactive Fuzzy Numbers}

Parameters such as rates or initial conditions in biomathematical models are intrinsically endowed with uncertainty. What follows is motivated by a particular model, that is, Malthus' model with decay [2]. In this model, $w+x_{0}=2$ or, similarly, $(w-1)+\left(x_{0}-1\right)=0$, where $w$ and $x_{0}$ are uncertain parameters that stand respectively for the decay rate and the initial condition. An approach for dealing with this model is given by treating the uncertain terms $(w-1)$ and $\left(x_{0}-1\right)$ as fuzzy numbers $A_{1}$ and $A_{2}$, respectively.

Let us start with a simple general example that represents the following situation: the sum of two fuzzy numbers "around to zero" equals another fuzzy number "around to zero". Let $+: \mathbb{R}^{2} \rightarrow \mathbb{R}$ be the function that associates each pair of real numbers $\left(x_{1}, x_{2}\right)$ with the sum $x_{1}+x_{2}$, that is: $+\left(x_{1}, x_{2}\right)=x_{1}+x_{2}$. Furthermore, let $A_{1}$ and $A_{2}$ be equal to the triangular fuzzy number $(-1 ; 0 ; 1)$. Note that $(-1 ; 0 ; 1)$ can be used to model the concept 'arround to zero'.

Different choices of a t-norm-based joint possibility distribution of $\left(A_{1}, A_{2}\right)$, that is, different choices of a t-norm, yield different extensions of + at $\left(A_{1}, A_{2}\right)$. For notational convenience, we simply write $\left(A_{1}+{ }_{J} A_{2}\right)$ instead of $+_{J}\left(A_{1}, A_{2}\right)$. Let $t_{D}$ denote respectively the drastic t-norm. Moreover, let $J_{t}$ denote the $t$-based joint possibility distribuitions of $A_{1}$ and $A_{2}$ for any t-norm $t$. In particular, the symbols $J_{D}$ and $J_{\wedge}$ stand respectively for the $t_{D}$-based and $\wedge$-based joint possibility distribuitions of $A_{1}$ and $A_{2}$. Note that $J_{D} \subseteq J_{t} \subseteq J_{\wedge}$ since $a t_{D} b \leq a t b \leq a \wedge b$ for all $a, b \in[0,1]$. Consequently, the norm of the sup- $J_{t}$ extension of any function $f$ is bounded by the norms of the sup- $J_{D}$ extension and the sup- $J_{\wedge}$ extension of $f$. In other words, for any function $f$, the following property holds:

$$
\left\|f_{J_{D}}\left(A_{1}, A_{2}\right)\right\| \leq\left\|f_{J_{t}}\left(A_{1}, A_{2}\right)\right\| \leq\left\|f_{J_{\wedge}}\left(A_{1}, A_{2}\right)\right\| .
$$

These inequalities can be verified by writing the sup-J extensions above in fuzzy relational equation form as in Eq. (9) and by using the sup-t composition's order-preserving property and the definition of the norm for fuzzy numbers. Hence, if we employ a t-norm-based joint possibility distribution $J_{t}$ to extend the function of addition, then we have that the norm of the resulting $\sup -J_{t}$ extension lies in the interval $[1,2]$ since $\left(A_{1}+{ }_{J_{D}} A_{2}\right)=(-1 ; 0 ; 1)$ and $\left(A_{1}+{ }_{J_{\wedge}} A_{2}\right)=(-2 ; 0 ; 2)$.

Extensions of + at $\left(A_{1}, A_{2}\right)$ with norm less than 1 can be obtained if we use other forms of joint possibility distributions that do not employ tnorms. This is the case for the completely correlated fuzzy numbers introduced by Fuller and Majlender $[14,10]$. Indeed, in our example, the triangular fuzzy numbers $A_{1}$ and $A_{2}$ are completely negatively correlated fuzzy numbers if their joint possibility relation $J$ equals $J_{-1,0}$ (cf. Equation 11). Note that $J_{-1,0}$ is a joint possibility distribution for $A_{1}$ and $A_{2}$ that is not t-norm based. Figure 1 exhibits the joint possibility distribution $J_{-1,0}$ for $A_{1}$ and $A_{2}$.

By definition, the sup- $J_{-1,0}$ extension of $f$ at 


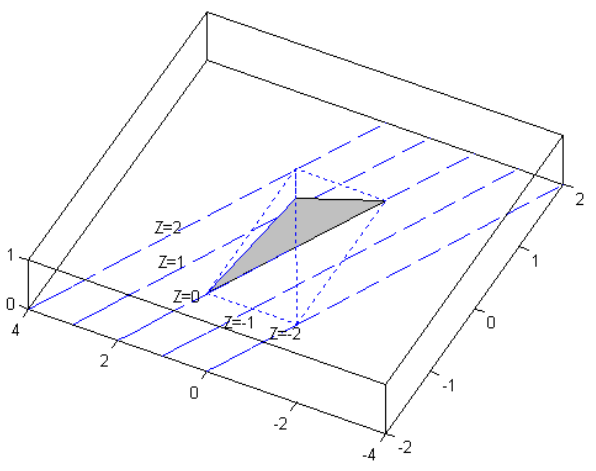

Figure 1: The joint possibility distribution $J_{-1,0}$ of $A_{1}$ and $A_{2}$ given by Equation (11) is depicted in gray. The dashed lines correspond to the level sets of the function $+(x, y)=z$ for $z=-2, \ldots, 2$.

$$
\begin{aligned}
& \left(A_{1}, A_{2}\right) \text { is } \\
& \quad\left(A_{1}+J_{-1,0} A_{2}\right)(z)=\bigvee_{z=x+y} A_{1}(x) \chi_{\{-x=y\}}(x, y) .
\end{aligned}
$$

An application of the Nguyen's theorem [15] leads to an elegant formula for the $\alpha$-cuts of $\left(A_{1}+{ }_{J_{-1,0}} A_{2}\right)$ :

$$
\left[\left(A_{1}+J_{-1,0} A_{2}\right)\right]^{\alpha}=(q+1)\left[A_{1}\right]^{\alpha}+r=0\left[A_{1}\right] \alpha=\{0\}
$$

for all $\alpha \in[0,1]$. Hence, using the representation theorem, we obtain that $\left(A_{1}+J_{-1,0} A_{2}\right)=\chi_{\{0\}}$, that is, the crisp number 0 , which implies that $\left\|\left(A_{1}+J_{-1,0} A_{2}\right)\right\|=0$.

The above example reveals that if we restrict ourselves to the t-norm based extension principle, then we may not be able to produce an extension of + at a pair $(A, B)$ such that $\left(A+J_{t} B\right)$ has a minimal norm. In fact, every pair of fuzzy numbers $A, B \notin \mathcal{P}(\mathbb{R})$ (that do not represent real numbers) has an infinite number of joint possibility distributions. Hence, there are infinitely many possible extensions of the sum operator at $(A, B)$.

The previous example shows that is possible to generate extensions of the sum operator at $\left(A_{1}, A_{2}\right)$ with different norms ranging from 0 to 2 . This observations leads us to the following question: Is it possible to control the norm of the sum of two fuzzy numbers by means of the sup-J extension principle? More precisely, is it possible to construct a sequence of joint possibility distribution for any pair of fuzzy numbers such that the norm of the corresponding extensions is increasing? In the next section, we will provide affirmative answers to these questions.

\section{A Family of Joint Possibility Distributions}

First of all, given a pair of fuzzy numbers, we will present a parametrized formula which leads to a family of fuzzy relations in $\mathbb{R}^{2}$ and then we will prove that they are indeed joint possibility distributions for the given pair of fuzzy numbers.
Since we want to have some control over the underlying interactivity, the proposed formula is endowed with a tunable parameter $\gamma \in[0,1]$ that plays the role of adjusting the norms of the corresponding sup-J extensions of the sum operator.

Given arbitrary $A_{1}, A_{2} \in \mathbb{R}_{\mathcal{F}}$, we define the functions $f_{\wedge}^{i}, f_{\vee}^{i}: \mathbb{R} \rightarrow \mathbb{R}$, where $i=1,2$, as follows:

$$
f_{\wedge}^{i}(z)=\bigwedge_{w \in\left[A_{3-i}\right]^{A_{i}(z)}}|w+z| \forall z \in \mathbb{R}
$$

and

$$
f_{\bigvee}^{i}(z)=\bigvee_{w \in\left[A_{3-i}\right]^{A_{i}(z)}}|w+z| \forall z \in \mathbb{R}
$$

Now, we are ready to define the following family $\left\{J_{\gamma}\right\}$ of fuzzy relations of $\mathbb{R}^{2}$ for $\gamma \in[0,1]$ :

$J_{\gamma}\left(x_{1}, x_{2}\right)=\left\{\begin{array}{cl}\bigwedge_{i=1}^{2} A_{i}\left(x_{i}\right) & ,\left|x_{1}+x_{2}\right| \leq v_{\gamma}\left(x_{1}, x_{2}\right) \\ 0 & , \text { otherwise }\end{array}\right.$

where

$$
v_{\gamma}\left(x_{1}, x_{2}\right)=\bigvee_{i=1}^{2}\left[(1-\gamma) f_{\wedge}^{i}\left(x_{i}\right)+\gamma f_{\vee}^{i}\left(x_{i}\right)\right] .
$$

Let us begin our investigation by showing that Equation (15) is a joint possibility distribution of $A_{1}$ and $A_{2}$ for every $\gamma \in[0,1]$. On the one hand, the inequality $J_{\gamma}(x, y) \leq A_{1}(x)$ holds for all $x, y \in \mathbb{R}$ from Equation (15), which implies that $\Pi_{J_{\gamma}}^{1}(x) \leq A_{1}(x)$. On the other hand, Proposition 1 guarantees that $\left[A_{i}\right]^{\alpha}, i=1,2$, are closed intervals for all $\alpha \in[0,1]$. Thus, an application of Weierstrass's theorem extreme value theorem ensures that there exists $x_{\wedge} \in$ $\left[A_{2}\right]^{A_{1}(x)}$ such that $\left|x+x_{\wedge}\right|=f_{\wedge}^{1}(x) \leq v_{\gamma}(x, y)$ for all $y \in \mathbb{R}$. The last observations implies that $\Pi_{J_{\gamma}}^{1}(x) \geq\left[A_{1}(x) \wedge A_{2}\left(x_{\wedge}\right)\right]=J_{\gamma}\left(x, x_{\wedge}\right)=A_{1}(x)$. Hence, we have that $\Pi_{J_{\gamma}}^{1}(x)=A_{1}(x)$ for all $x \in \mathbb{R}$. Similarly, we can show that $\Pi_{J_{\gamma}}^{2}(y)=A_{2}(y)$ for all $y \in \mathbb{R}$. Consequently, we obtain the next theorem.

Theorem 1 Given $A_{1}, A_{2} \in \mathbb{R}_{\mathcal{F}}$, the fuzzy relation $J_{\gamma}$ defined as in Equation (15) is a joint possibility distribution of $A_{1}$ and $A_{2}$ for every $\gamma \in[0,1]$.

For $0 \leq \gamma_{1} \leq \gamma_{2} \leq 1$, we have that $J_{\gamma_{1}} \subseteq J_{\gamma_{2}}$ since $v_{\gamma_{1}}(x, y) \leq v_{\gamma_{2}}(x, y)$ for all $x, y \in \mathbb{R}^{2}$. Thus, the bigger the parameter $\gamma$, the greater the fuzzy set given by means of Equation (15) with respect to the inclusion order. This is actually what we were looking for. Writing the corresponding sup-J extensions in terms of fuzzy relational compositions (cf. Equation (9)) and using the fact that the supmin composition is an order-preserving operator, we have that

$$
\begin{gathered}
R_{f} \circ J_{\gamma_{1}} \subseteq R_{f} \circ J_{\gamma_{2}} \text { if } \gamma_{1} \leq \gamma_{2} \\
\Rightarrow\left\|\left(A_{1}+J_{\gamma_{1}} A_{2}\right)\right\| \leq\left\|\left(A_{1}+J_{\gamma_{2}} A_{2}\right)\right\| \text { if } \gamma_{1} \leq \gamma_{2} .
\end{gathered}
$$


Thus, the norm of the sup- $J_{\gamma}$ extension of the sum of two fuzzy numbers can be adjusted by modifying the parameter $\gamma$.

Note that $J_{1}=J_{\wedge}$ for all $A_{1}, A_{2} \in \mathbb{R}_{\mathcal{F}}$ due to the following observations. Consider arbitrary $x \in$ $\left[A_{1}\right]^{0}$ and $y \in\left[A_{2}\right]^{0}$ and let us suppose, without loss of generality, that $A_{1}(x) \leq A_{2}(y)$. In this case, we have that $y \in\left[A_{2}\right]^{A_{1}(x)}$ and consequently $\mid x+$ $y \mid \leq f_{\vee}^{1}(x) \leq v_{1}(x, y)=f_{\vee}^{1}(x) \vee f_{\vee}^{2}(y)$. Hence, the equality $J_{1}(x, y)=J_{\wedge}(x, y)$ follows from Equation (15).

Let us finish this section by constructing some joint possibility distributions using our simple example. Henceforth, let $A_{1}=A_{2}=(-1 ; 0 ; 1)$ and $f(x, y)=x+y$ for all $x, y \in \mathbb{R}$.

As mentioned before, $\left\|\left(A_{1}+J_{\gamma} A_{2}\right)\right\|$ is increasing with respect to $\gamma$. Let us now express $\left\|\left(A_{1}+{ }_{J_{\gamma}} A_{2}\right)\right\|$ in terms of a simple formula. Specifically, we will show that $\left\|\left(A_{1}+J_{\gamma} A_{2}\right)\right\|=2 \gamma$.

On the one hand, $f_{\wedge}^{i}(z)=0$ for all $z \in\left[A_{i}\right]^{0}$ since we have that $[-|z|,|z|] \subseteq\left[A_{3-i}\right]^{A_{i}(z)}$ because $\left[A_{3-i}\right]^{A_{i}(z)}$ is a closed interval (see Prop. 1) and $A_{3-i}(z)=A_{3-i}(-z)=A_{i}(z)$ for $i=1,2$. Moreover, we have that $f_{\vee}^{i}(z) \leq 2$ for all $z \in\left[A_{i}\right]^{0}$ because $2=\bigvee\left\{|z+w| \mid z \in\left[A_{1}\right]^{0}=[-1,1], w \in\right.$ $\left.\left[A_{2}\right]^{0}=[-1,1]\right\}$ and $\left[A_{i}\right]^{\beta} \subseteq\left[A_{i}\right]^{0}$ for all $\beta$ in $[0,1]$ and $i=1,2$ by the second property of Proposition 1. The last observation implies that $v_{\gamma}(x, y) \leq 2 \gamma$ for all $(x, y) \in\left[A_{1}\right]^{0} \times\left[A_{2}\right]^{0}$. Thus, for any $z$ such that $\left(A_{1}+J_{\gamma} A_{2}\right)(z)>0$, there exist $(x, y) \in\left[J_{\gamma}\right]^{\beta}$, $\left(A_{1}+J_{\gamma} A_{2}\right)(z) \geq \beta>0$, satisfying $x+y=z$, and therefore $|x+y|=|z| \leq v_{\gamma}(x, y) \leq 2 \gamma$. The inequality $|x+y| \leq 2 \gamma$ implies that $\left[\left(A_{1}+J_{\gamma} A_{2}\right)\right]^{\beta} \subseteq$ $[-2 \gamma, 2 \gamma]$ for all $\beta \in(0,1]$ and therefore we obtain $\left[\left(A_{1}+J_{\gamma} A_{2}\right)\right]^{0} \subseteq[-2 \gamma, 2 \gamma]$ from the fourth property of Proposition 1. Hence, $\left\|\left(A_{1}+J_{\gamma} A_{2}\right)\right\| \leq 2 \gamma$.

On the other hand, $\left[A_{3-i}\right]^{A_{i}(z)}$ is a closed interval by Proposition 1 and an application of the intermediate value theorem ensures that there exist $c_{z}^{i} \in$ $\left[A_{3-i}\right]^{A_{i}(z)}$ such that $\left|c_{z}^{i}+z\right|=\gamma f_{\vee}^{i}(z)$ for all $z \in \mathbb{R}$ and $i=1,2$. Moreover, $f_{\vee}^{i}(z)=|z+z|$ for all $z \in$ $\left[A_{i}\right]^{0}$ and $i=1,2$ since we have that $\left[A_{3-i}\right]^{A_{i}(z)}=$ $[-|z|,|z|]$ by the definition of the $\alpha$-cuts of $A_{1}=$ $A_{2}=(-1 ; 0 ; 1)$. This implies that $v_{\gamma}(z, z)=\gamma|z+z|$ for all $z \in\left[A_{1}\right]^{0}=[-1,1]$. If $x_{1}^{(n)}=1-\frac{1}{n} \in$ $\left[A_{1}\right]^{\frac{1}{n}}$, then there exists $x_{2}^{(n)} \in\left[A_{2}\right]^{A_{1}\left(x_{1}^{(n)}\right)}$ such that $\left|x_{1}^{(n)}+x_{2}^{(n)}\right|=\gamma f_{\vee}^{1}\left(x_{1}^{(n)}\right) \leq v_{\gamma}\left(x_{1}^{(n)}, x_{2}^{(n)}\right)$ which implies that $\left(A_{1}+J_{\gamma} A_{2}\right)\left(x_{1}^{(n)}+x_{2}^{(n)}\right) \geq \frac{1}{n}$. Taking the limit of $\left|x_{1}^{(n)}+x_{2}^{(n)}\right|=\gamma f_{\vee}^{1}\left(x_{1}^{(n)}\right)=2 \gamma\left|x_{1}^{(n)}\right|$ we obtain that $\left\|\left(A_{1}+J_{\gamma} A_{2}\right)\right\| \geq 2 \gamma$. Therefore, the following formula holds:

$$
\left\|\left(A_{1}+{ }_{J_{\gamma}} A_{2}\right)\right\|=2 \gamma .
$$

For this particular example, our approach yields a one-to-one continuous increasing mapping that associates each $\gamma \in[0,1]$ with the norm of the sup$J_{\gamma}$ extension, that is, $\left\|\left(A_{1}+J_{\gamma} A_{2}\right)\right\|$. Moreover, $\left\|\left(A_{1}+J_{0} A_{2}\right)\right\|$ and $\left\|\left(A_{1}+J_{1} A_{2}\right)\right\|$ constitute respectively the minimal and maximal norms that can be obtained by means of a sup- $J_{\gamma}$ extension of the sum operator at $\left(A_{1}, A_{2}\right)$. Recall that the norm of the sup-J extension based on a t-norm-based joint possibility distribution has 1 as a lower bounded. Thus, our approach provides a way to model the interactivity of $A_{1}$ and $A_{2}$ in terms of sup-J extensions such that the resulting expressions of sums have norms between 0 and 1 .

Figure 2 displays a lateral view of the joint possibility distribution $J_{0.5}$. Figures 3 and 4 depict a top-down view of the joint possibility distribution $J_{0.25}$ and $J_{0.75}$.

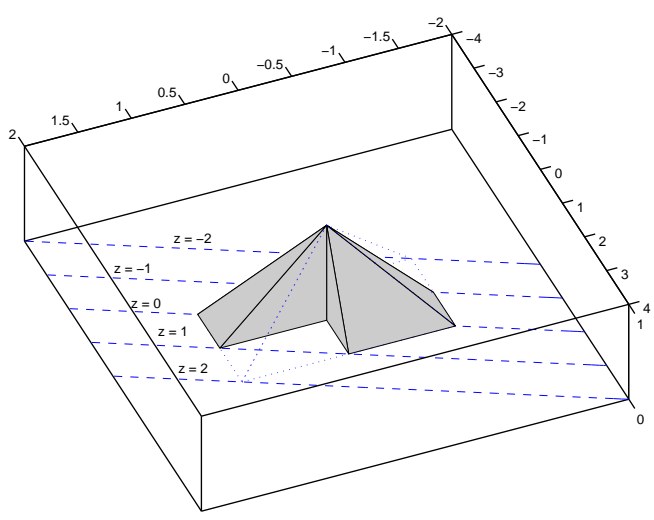

Figure 2: The joint possibility distribution $J_{0.5}$ of $A_{1}=(-1 ; 0 ; 1)$ and $A_{2}=(-1 ; 0 ; 1)$ is depicted in the gray area. The sketch of $J_{1}$ is given by the dotted line. The dashed lines correspond to the level sets of the function $+(x, y)=z$ for $z=-2, \ldots, 2$.

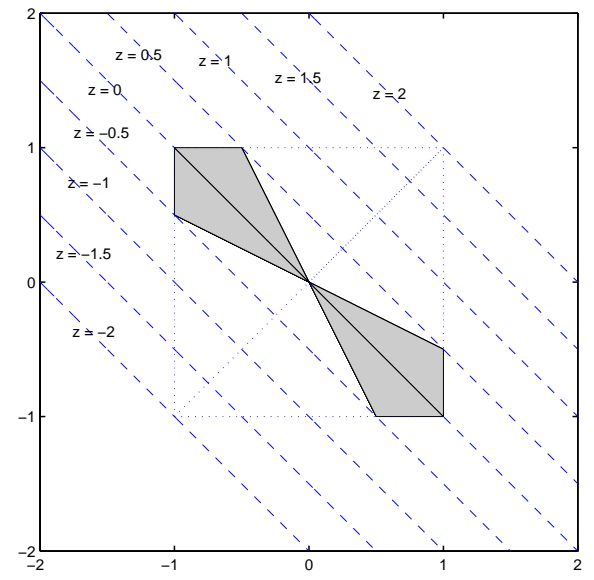

Figure 3: The joint possibility distribution $J_{0.25}$ of $A_{1}=(-1 ; 0 ; 1)$ and $A_{2}=(-1 ; 0 ; 1)$ is depicted in the gray area. The sketch of $J_{1}$ is given by the dotted line. The dashed lines correspond to the level sets of the function $f(x, y)=z$ for $z=-2,-1.5, \ldots, 1.5,2$.

Recall that $\left\|\left(A_{1}+J_{-1,0} A_{2}\right)\right\|=\left\|\left(A_{1}+J_{0} A_{2}\right)\right\|=$ 0 . These equalities suggest that $J_{0}$ coincides with $J_{-1,0}$ since the unique fuzzy number that has norm 0 is the crisp number 0 (i.e., $\chi_{\{0\}}$ ). On the one hand, $\chi_{-u=v}(x, y)=1$ if and only if $0=x+y$, and therefore we have that $J_{-1,0}(x, y)=A_{1}(x)$ if 


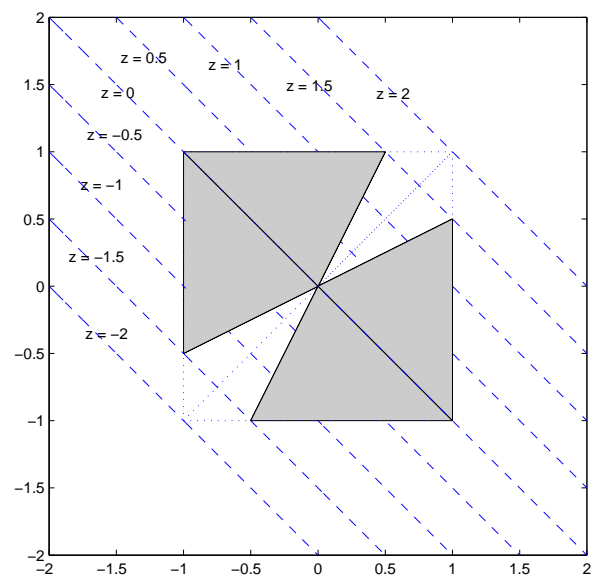

Figure 4: The joint possibility distribution $J_{0.75}$ of $A_{1}=(-1 ; 0 ; 1)$ and $A_{2}=(-1 ; 0 ; 1)$ is depicted in the gray area. The sketch of $J_{1}$ is given by the dotted line. The dashed lines correspond to the level sets of the function $f(x, y)=z$ for $z=-2,-1.5, \ldots, 1.5,2$.

$x+y=0$ and otherwise $J_{-1,0}(x, y)$ equals 0 . On the other hand, $\left[A_{1}\right]^{\alpha}=\left[A_{2}\right]^{\alpha}$ for all $\alpha \in[0,1]$ since $A_{1}=A_{2}$. We conclude that $f_{\wedge}^{i}(x)=0$ for all $x \in \mathbb{R}$ and $i=1,2$. Equation (15) and the last observation imply that $J_{0}(x, y)=A_{1}(x) \wedge A_{2}(x)=A_{1}(x)$ if $x+y=0$. Otherwise, we have that $J_{0}(x, y)=$ 0 . To summarize, we have that $J_{0}=J_{-1,0}$ in this example.

Finally, we would like to point out that there are joint possibility distributions $J_{q, r}$ of completely correlated fuzzy numbers that differ from all joint possibility distributions $J_{\gamma}$ where $\gamma \in[0,1]$. For example, the joint possibility distribution $J_{-1,1}$ of $(-1 ; 0 ; 1)$ and $(0 ; 1 ; 2)$ differs from every joint possibility distribution $J_{\gamma}$ of these triangular fuzzy numbers.

\section{Concluding Remarks}

Fuller and Majlender's definition of completely correlated fuzzy numbers can only be applied to a subset of the pairs of fuzzy numbers in which a joint possibility distribution can be generated in terms a characteristic function of a line. In contrast, our approach as well as the t-norm-based approach can be applied to establish an interactivity relation between any pair of fuzzy numbers.

This paper shows that our approach generates a family of joint possibility functions, denoted by $J_{\gamma}$ such that the norm of the sup- $J_{\gamma}$ extension represents an increasing mapping with respect the parameter $\gamma$. This property allows us to determine the effect of the underlying interactivity on the sup$J$ extension of the sum operation in terms of the norm. We considered a specific example and showed that, in this particular case, the norm of the sup$J_{\gamma}$ extension of two fuzzy numbers is given by $2 \gamma$ with $\gamma \in[0,1]$ whereas the t-norm-based approach produces extensions whose norms are at least 1 .

\section{References}

[1] J. Baetens and B. De Baets. In Proceedings of the joint 2009 International Fuzzy Systems Association world congress and 2009 European Society for Fuzzy Logic and Technology conference.

[2] V.M. Cabral and L.C. Barros. Fuzzy differential equation with completely correlated parameters. Fuzzy Sets and Systems, 2014. available online.

[3] L. A. Zadeh. Fuzzy sets. Information and Control, 8(3):338-353, 1965.

[4] G. Birkhoff. Lattice Theory. American Mathematical Society, Providence, 3rd, edition, 1993.

[5] A. Di Nola, S. Sessa, W. Pedrycz, and E. Sanchez. Fuzzy Relation Equations and Their Applications to Knowledge Engineering. Kluwer Academic Publishers, Norwell, MA, 1989.

[6] G. J. Klir and B. Yuan. Fuzzy Sets and Fuzzy Logic; Theory and Applications. Prentice Hall, Upper Saddle River, N. Y., 1995.

[7] W. Pedrycz and F. Gomide. Fuzzy Systems Engineering: Towards Human-Centric Computing. Wiley, IEEE Press, New York, 2007.

[8] C.V. Negoiţă and D.A. Ralescu. Applications of fuzzy sets to systems analysis. Interdisciplinary systems research. Wiley, 1975.

[9] L. A. Zadeh. The concept of a linguistic variable and its application to approximate reasoning - I. Information Sciences, 8:199-249, 1975.

[10] R. Fullér and P. Majlender. On interactive fuzzy numbers. Fuzzy Sets and Systems, 143(3):355 - 369, 2004.

[11] R. Fullér. On generalization of nguyen's theorem: A short survey of recent developments. In János Fodor and Robert Fullér, editors, $A d$ vances in Soft Computing, Intelligent Robotics and Control, volume 8 of Topics in Intelligent Engineering and Informatics, pages 183-190. Springer International Publishing, 2014.

[12] B. Bede. Mathematics of Fuzzy Sets and Fuzzy Logic, volume 295 of Studies in Fuzziness and Soft Computing. Springer, 2013.

[13] R. Fullér and T. Keresztfalvi. On generalization of Nguyen's theorem. Fuzzy Sets and Systems, 41(3):371 - 374, 1991.

[14] C. Carlsson, R. Fuller, and P. Maj lender. Additions of completely correlated fuzzy numbers. In Fuzzy Systems, 2004. Proceedings. 2004 IEEE International Conference on, volume 1, pages 535-539, July 2004.

[15] Hung T Nguyen. A note on the extension principle for fuzzy sets. Journal of Mathematical Analysis and Applications, 64(2):369 - 380, 1978. 